\title{
Time-dependent pseudo-Hermitian Hamiltonians defining a unitary quantum system and uniqueness of the metric operator
}

\author{
Ali Mostafazadeh \\ Department of Mathematics, Koç University, 34450 Sariyer, Istanbul, Turkey \\ Received 27 February 2007; accepted 30 April 2007 \\ Available online 6 May 2007 \\ Editor: J.-P. Blaizot
}

\begin{abstract}
The quantum measurement axiom dictates that physical observables and in particular the Hamiltonian must be diagonalizable and have a real spectrum. For a time-independent Hamiltonian (with a discrete spectrum) these conditions ensure the existence of a positive-definite inner product that renders the Hamiltonian self-adjoint. Unlike for a time-independent Hamiltonian, this does not imply the unitarity of the Schrödinger timeevolution for a general time-dependent Hamiltonian. We give an additional necessary and sufficient condition for the unitarity of time-evolution. In particular, we obtain the general form of a two-level Hamiltonian that fulfills this condition. We show that this condition is geometrical in nature and that it implies the reality of the adiabatic geometric phase angles. We also address the problem of the uniqueness of the metric operator.
\end{abstract}

(c) 2007 Elsevier B.V. All rights reserved.

PACS: 03.65.-w

Keywords: Pseudo-Hermitian; Unitary; Time-dependent Hamiltonian; $\mathcal{P} \mathcal{T}$-symmetry; Inner product; Metric operator; Geometric phase

\section{Introduction}

In quantum mechanics, the inner product of the Hilbert space of a quantum system is not an observable quantity. Because all (separable) Hilbert spaces are unitary equivalent, a convenient choice is to fix the inner product to be the $L^{2}$-inner product $\langle\cdot \mid \cdot\rangle$ and formulate the theory on the resulting Hilbert space $\mathcal{H}$. In recent years, it has become clear that one can also formulate a consistent quantum theory that employs certain non-self-adjoint Hamiltonian operators $H: \mathcal{H} \rightarrow \mathcal{H}$, [1-3]. The latter are operators that can be made self-adjoint, if one selects a new inner product $\langle\cdot, \cdot\rangle_{+}$. This in turn implies that $H$ is a diagonalizable operator with a real spectrum. ${ }^{1}$ The physical Hilbert space $\mathcal{H}_{\text {phys }}$ is obtained by endowing the span of the eigenvectors of $H$ with the inner product $\langle\cdot, \cdot\rangle_{+}$and completing the resulting inner product space [4]. The observables are identified with

E-mail address: amostafazadeh@ku.edu.tr.

1 The diagonalizability of $H$ means that it has a complete set of eigenvectors. self-adjoint operators acting in $\mathcal{H}_{\text {phys }},[4,5] .{ }^{2}$ The purpose of this Letter is to show that the above mentioned developments do not directly extend to diagonalizable time-dependent Hamiltonians having a real spectrum.

Time-dependent Hamiltonian operators have many applications in non-relativistic quantum mechanics. They are also indispensable in the Hamiltonian formulation of quantum field theories. Therefore a consistent treatment of pseudo-Hermitian (and in particular $\mathcal{P} \mathcal{T}$-symmetric [6]) quantum field theories calls for a careful study of the extension of the methods of pseudo-Hermitian quantum mechanics [5] to time-dependent Hamiltonians. Such Hamiltonians arise naturally in quantum cosmological applications of pseudo-Hermitian quantum mechanics [7].

In [8] the authors consider particular examples of timedependent pseudo-Hermitian Hamiltonians that admit a time-

\footnotetext{
2 These constructions do not lead to a generalization of quantum mechanics but to another of its equivalent representations. This is because one can describe the very same systems using self-adjoint Hamiltonians and self-adjoint observables acting in $\mathcal{H}$, [4].
} 
independent (positive-definite) metric operator. In this article, we will refer to such Hamiltonians as quasi-stationary and show that a direct extension of the methods of pseudo-Hermitian quantum mechanics to time-dependent Hamiltonians is possible provided that they are quasi-stationary. We will then derive a necessary and sufficient condition under which a given time-dependent diagonalizable operator with a real and discrete spectrum is quasi-stationary.

In the remainder of this section we give a brief review of the spectral methods used in the construction of the metric operators for a diagonalizable operator with a real and discrete spectrum $[2,9]$.

Let $\mathcal{H}$ be a separable (reference) Hilbert space with $\left(L^{2}-\right)$ inner product $\langle\cdot \mid \cdot\rangle$, and $H: \mathcal{H} \rightarrow \mathcal{H}$ be a diagonalizable (Hamiltonian) operator with a real and discrete spectrum. The diagonalizability of $H$ and the reality of its spectrum are necessary conditions for the applicability of the standard quantum measurement theory [10]. The discreteness of the spectrum of $H$ is a simplifying assumption that could be relaxed depending on the particular operator in question $[7,11]$.

As shown in [9], an operator $H$ with the above-mentioned properties is necessarily pseudo-Hermitian, i.e., there is a Hermitian invertible (pseudo-metric) operator $\eta: \mathcal{H} \rightarrow \mathcal{H}$ satisfying

$H^{\dagger}=\eta H \eta^{-1}$.

Furthermore, among the infinity of pseudo-metric operators $\eta$ satisfying this condition there are positive-definite operators $\eta_{+}$that can be used to construct a positive-definite inner product $[2],{ }^{3}$

$\langle\cdot, \cdot\rangle_{+}:=\left\langle\cdot \mid \eta_{+} \cdot\right\rangle$

In view of this relation and the $\eta_{+}$-pseudo-Hermiticity of $H$, i.e.,

$H^{\dagger}=\eta_{+} H \eta_{+}^{-1}$,

$H$ is self-adjoint with respect to the inner product $\langle\cdot, \cdot\rangle_{+}$. The operator $\eta_{+}$and the corresponding inner product $\langle\cdot, \cdot\rangle_{+}$that defines the physical Hilbert space $\mathcal{H}_{\text {phys }}$ of the system are not unique [16]. ${ }^{4}$

An important observation made in [2] is that $H$ is related to a Hermitian operator $h: \mathcal{H} \rightarrow \mathcal{H}$ via a similarity transformation. For example, we can choose $\eta_{+}^{1 / 2}$ to perform such a similarity

\footnotetext{
${ }^{3}$ Eq. (1) was initially considered by Pauli [12] in trying to formalize an idea due to Dirac [13] that later led to the development of the indefinite-metric quantum theories [14]. But Pauli and others who contributed to this development only considered the case that $\eta$ was a fixed (given) indefinite operator. The idea of treating (1) as an equation for $\eta$ and realizing that for some $H$ one can choose a positive-definite operator among all possible $\eta$ 's, that is embraced in [2], has its origin in the particular definition of pseudo-Hermiticity given in [9]. This is different from the old notion of "pseudo-Hermiticity" used in indefinite-metric theories. The latter is known as $J$-Hermiticity in mathematical literature [15]. For a detailed discussion see [10] and reference 1 therein.

4 The so-called $\mathcal{C P} \mathcal{T}$-inner products $[3,17,18]$ that can be constructed for typical $\mathcal{P} \mathcal{T}$-symmetric Hamiltonians form a special class of the inner products $\langle\cdot, \cdot\rangle_{+},[19]$.
}

transformation and define $h$ as

$h:=\eta_{+}^{1 / 2} H \eta_{+}^{-1 / 2}$.

In fact, viewing $H$ and $h$ as acting in $\mathcal{H}_{\text {phys }}$ and $\mathcal{H}$ respectively and viewing $\eta_{+}^{1 / 2}$ as an operator mapping $\mathcal{H}_{\text {phys }}$ to $\mathcal{H}$, we find that indeed $\eta_{+}^{1 / 2}$ is a unitary operator, ${ }^{5}$ and $h$ and $H$ are unitary equivalent [4]. This in turn allows for the formulation of the theory in terms of the Hermitian Hamiltonian $h$ within the framework of conventional quantum mechanics. ${ }^{6}$

Because $H$ is assumed to be diagonalizable, one can construct a complete biorthonormal system $\left\{\psi_{n}, \phi_{n}\right\}$ for the Hilbert space such that $\psi_{n}$ and $\phi_{n}$ are respectively the eigenvectors of $H$ and $H^{\dagger}$ with eigenvalue $E_{n}$, [2],

$H\left|\psi_{n}\right\rangle=E_{n}\left|\psi_{n}\right\rangle, \quad H^{\dagger}\left|\phi_{n}\right\rangle=E_{n}\left|\phi_{n}\right\rangle$.

We also recall that "biorthonormality" means

$\left\langle\phi_{n} \mid \psi_{m}\right\rangle=\delta_{m n}, \quad \sum_{n}\left|\psi_{n}\right\rangle\left\langle\phi_{n}\right|=1$,

where we use Dirac's bra-ket notation in $\mathcal{H}, \delta_{m n}$ stands for the Kronecker delta symbol, and 1 denotes the identity operator. In terms of the biorthonormal system $\left\{\psi_{n}, \phi_{n}\right\}$, we can construct the following metric operator.

$\eta_{+}=\sum_{n}\left|\phi_{n}\right\rangle\left\langle\phi_{n}\right|$.

Indeed every metric operator that satisfies (3) can be expressed in this form for some biorthonormal system $\left\{\psi_{n}, \phi_{n}\right\}$ fulfilling (5), [19].

\section{Quasi-stationary pseudo-Hermitian Hamiltonians}

Consider a Hamiltonian operator $H[R]$ that is parameterized by points $R$ of a parameter space $M$. Suppose that $H[R]$ is diagonalizable and has a real and discrete spectrum for all $R \in M$. Then there is an $R$-dependent biorthonormal system $\left\{\psi_{n}, \phi_{n}\right\}$ satisfying (5) with $H=H[R]$ for all $R \in M$. Furthermore, $H[R]$ is $\eta_{+}$-pseudo-Hermitian for a metric operator $\eta_{+}$ of the form (7). We will use the following notation to make the $R$-dependence of $\psi_{n}, \phi_{n}$ and $\eta_{+}$explicit: $\left|\psi_{n}, R\right\rangle:=\left|\psi_{n}\right\rangle$, $\left|\phi_{n}, R\right\rangle:=\left|\phi_{n}\right\rangle$, and $\eta_{+}[R]:=\eta_{+}$.

Next, let $T \in \mathbb{R}^{+}$, and $\gamma:[0, T] \rightarrow M$ be a smooth curve in $M$ that determines the time-dependence of the parameters $R$ and the Hamiltonian according to $R(t):=\gamma(t)$ and $H(t):=H[R(t)]$, respectively. Let us also introduce the abbreviated notation: $\left|\psi_{n}, t\right\rangle:=\left|\psi_{n}, R(t)\right\rangle,\left|\phi_{n}, t\right\rangle:=\left|\phi_{n}, R(t)\right\rangle$, and $\eta_{+}(t):=\eta_{+}[R(t)]$.

As pointed out in [7], the $\eta_{+}(t)$-pseudo-Hermiticity of $H(t)$ does not generally ensure the unitarity of the Schrödinger timeevolution determined by $H(t)$ even if we define the Hilbert

\footnotetext{
5 This means that for all $\psi, \phi \in \mathcal{H}_{\text {phys }},\left\langle\eta^{1 / 2} \psi \mid \eta^{1 / 2} \phi\right\rangle=\langle\psi, \phi\rangle_{+}$.

${ }^{6}$ For the cases that $\mathcal{H}$ is infinite-dimensional and $H$ has the standard (kinetic + potential) form, the equivalent Hermitian Hamiltonian $h$ is a typically nonlocal operator $[5,11]$. But there are specific cases that it turns out to be local [20].
} 
space using the inner product $\langle\cdot, \cdot\rangle_{+}:=\left\langle\cdot \mid \eta_{+}(t) \cdot\right\rangle$. To see this, we denote the time-evolution operator of the system by $U(t)$, i.e., the operator satisfying the defining relations:

$i \hbar \frac{d}{d t} U(t)=H(t) U(t), \quad U(0)=1$.

Let $\prec \cdot, \cdot \succ$ be a general possibly time-dependent positivedefinite inner product on $\mathcal{H}$. Then we can always express $\prec \cdot, \succ \succ$ in terms of a possibly time-dependent metric operator $\xi_{+}(t)$ according to [21]

$\prec \cdot, \succ=\left\langle\cdot \mid \xi_{+}(t) \cdot\right\rangle$.

Suppose that $\psi(t)$ and $\phi(t)$ are arbitrary evolving state vectors;

$\psi(t):=U(t) \psi(0), \quad \phi(t):=U(t) \phi(0)$.

Then the unitarity of time-evolution with respect to the inner product $\prec \cdot, \cdot \succ$ means that $\prec \psi(t), \phi(t) \succ$ does not depend on $t$. In view of (9) and (10), this condition is equivalent to

$\xi_{+}(t)=U(t)^{-1 \dagger} \xi_{+}(0) U(t)^{-1}$.

Differentiating both sides of this equation and using (8) we find

$H(t)^{\dagger}=\xi_{+}(t) H(t) \xi_{+}(t)^{-1}-i \xi(t) \dot{\xi}(t)^{-1}$,

where a dot denotes a time-derivative. Eq. (12) shows that $H(t)$ is $\xi_{+}$-pseudo-Hermitian if and only if $\xi_{+}$is time-independent.

The requirement of the unitarity of time-evolution demands that the inner product of the Hilbert space be defined by a metric operator fulfilling (12). On the other hand quantum measurement theory (projection axiom) requires $H(t)$ to be selfadjoint with respect to the defining inner product of the physical Hilbert space of the system. These two constraints imply that $a$ time-dependent Hamiltonian operator $H(t)$ defines a consistent unitary quantum system if and only if $H(t)$ is $\eta_{+}$-pseudoHermitian for a time-independent metric operator $\eta_{+}$. We will call such a Hamiltonian quasi-stationary.

Requiring $H(t)$ to be quasi-stationary puts a sever restriction on its eigenvectors. To see this we choose an arbitrary metric operator $\eta_{+}$satisfying (3), use an appropriate biorthonormal system $\left\{\left|\psi_{n}, t\right\rangle,\left|\phi_{n}, t\right\rangle\right\}$ to express it in the form (7), and demand that the time-derivative of both sides of this equation vanishes. In view of (6), this yields

$\mathcal{A}_{m n}(t)=\mathcal{A}_{n m}(t)^{*}$,

where

$\mathcal{A}_{m n}(t):=i\left\langle\phi_{m}, R\left|\frac{d}{d t}\right| \psi_{n}, R\right\rangle$.

If we assume that all the parameters $R$ describe physical situations, the condition (13) must be met for all possible curves $\gamma:[0, T] \rightarrow M$. This is equivalent to

$A_{m n}[R]=A_{n m}[R]^{*}$,

where

$A_{m n}[R]:=i\left\langle\phi_{m}, R|d| \psi_{n}, R\right\rangle:=\sum_{i=1}^{n} i\left\langle\phi_{m}, R\left|\frac{\partial}{\partial R_{i}}\right| \psi_{n}, R\right\rangle d R_{i}$.
The one-form $A_{n n}[R]$ is the Berry's connection one-form for a diagonalizable non-Hermitian Hamiltonian [22]. Therefore, a simple implication of (15) is that the adiabatic geometric phase angles for the system are real. ${ }^{7}$ This is actually to be expected, because the system admits a Hermitian representation in terms of a Hermitian Hamiltonian, and like other physical quantities the geometric phase angles can be calculated in the Hermitian representation where they are clearly real.

It is important to observe that the condition that $H(t)$ be quasi-stationary is equivalent to the requirement of the existence of a biorthonormal system $\left\{\left|\psi_{n}, R\right\rangle,\left|\phi_{n}, R\right\rangle\right\}$ such that $\left|\psi_{n}, R\right\rangle$ and $\left|\phi_{n}, R\right\rangle$ are respectively the eigenvectors of $H[R]$ and $H[R]^{\dagger}$ and that (15) is satisfied. Note also that this condition is not sensitive to the duration of the evolution of the system and is completely geometrical in nature.

\section{Two-level quasi-stationary pseudo-Hermitian Hamiltonians}

Consider the case that $\mathcal{H}$ is the two-dimensional complex vector space $\mathbb{C}^{2}$ endowed with the Euclidean $\left(L^{2}\right.$-) inner product: $\langle\psi \mid \phi\rangle:=\sum_{a=1}^{2} \psi^{a *} \phi^{a}$, where $\psi:=\left(\begin{array}{l}\psi^{1} \\ \psi^{2}\end{array}\right), \phi:=\left(\begin{array}{l}\phi^{1} \\ \phi^{2}\end{array}\right)$, and $\psi^{a}, \phi^{a} \in \mathbb{C}$ for all $a \in\{1,2\}$. In the standard basis $\left\{\left(\begin{array}{l}1 \\ 0\end{array}\right),\left(\begin{array}{l}0 \\ 1\end{array}\right)\right\}$ of $\mathbb{C}^{2}$, we can represent the most general diagonalizable Hamiltonian operator $H$ with a real spectrum as

$H=q I+H_{0}, \quad H_{0}=\left(\begin{array}{cc}\mathfrak{a} & \mathfrak{b} \\ \mathfrak{c} & -\mathfrak{a}\end{array}\right)$,

where $q \in \mathbb{R}, I$ is the $2 \times 2$ unit matrix, $\mathfrak{a}, \mathfrak{b}, \mathfrak{c} \in \mathbb{C}$, and $\mathfrak{a}^{2}+\mathfrak{b} \mathfrak{c}$ is real and nonnegative, $[16,23]$. The problem of finding the most general metric operator $\eta_{+}$for such a Hamiltonian has been completely solved in [23]. ${ }^{8}$

Reparameterizing $H_{0}$ in the form [25]

$H_{0}=E\left(\begin{array}{cc}\cos \theta & e^{-i \varphi} \sin \theta \\ e^{i \varphi} \sin \theta & -\cos \theta\end{array}\right)$,

where $E:=\sqrt{\mathfrak{a}^{2}+\mathfrak{b c}} \in[0, \infty), \theta, \varphi \in \mathbb{C}, \Re(\theta) \in[0, \pi],{ }^{9}$ and $\Re(\varphi) \in[0,2 \pi)$, we can express the most general biorthonormal system associated with $H$ as

$\left|\psi_{1}\right\rangle=\mathfrak{n}_{1}^{-1 *}\left(\begin{array}{c}\cos \frac{\theta}{2} \\ e^{i \varphi} \sin \frac{\theta}{2}\end{array}\right), \quad\left|\psi_{2}\right\rangle=\mathfrak{n}_{2}^{-1 *}\left(\begin{array}{c}\sin \frac{\theta}{2} \\ -e^{i \varphi} \cos \frac{\theta}{2}\end{array}\right)$,

$\left|\phi_{1}\right\rangle=\mathfrak{n}_{1}\left(\begin{array}{c}\cos \frac{\theta^{*}}{2} \\ e^{i \varphi^{*}} \sin \frac{\theta^{*}}{2}\end{array}\right), \quad\left|\phi_{2}\right\rangle=\mathfrak{n}_{2}\left(\begin{array}{c}\sin \frac{\theta^{*}}{2} \\ -e^{i \varphi^{*}} \cos \frac{\theta^{*}}{2}\end{array}\right)$,

where $\mathfrak{n}_{1}, \mathfrak{n}_{2} \in \mathbb{C}-\{0\}$ are arbitrary. The eigenvalues of $H$ (and $H^{\dagger}$ ) are given by $E_{1}=q+E$ and $E_{2}=q-E$. Substituting (20) in (7) and carrying out the necessary calculations, we find the following expression for the most general metric

\footnotetext{
7 The dynamical phase angles are also real, because $H(t)$ has a real spectrum

8 The two-level pseudo-Hermitian Hamiltonians have also been considered in $[4,18,24]$.

$9 \Re$ and $\Im$ stand for the real and imaginary part of their argument respectively.
} 
operator $\eta_{+}$such that $H$ is $\eta_{+}$-pseudo-Hermitian [23].

$\eta_{+}=k\left(\begin{array}{cc}a u+b & \lambda^{*} \\ \lambda & e^{2 \Im(\varphi)}(a+b u)\end{array}\right)$,

where $k:=\left|\mathfrak{n}_{2}\right|^{2}$ and $u:=\left|\mathfrak{n}_{1} / \mathfrak{n}_{2}\right|^{2}$ are arbitrary positive real parameters manifesting the non-uniqueness of $\eta_{+}$, and

$a:=\left|\cos \frac{\theta}{2}\right|^{2}, \quad b:=\left|\sin \frac{\theta}{2}\right|^{2}$,

$\lambda:=e^{i \varphi}\left(u \zeta^{*}-\zeta\right), \quad \zeta:=\sin \frac{\theta}{2} \cos \frac{\theta^{*}}{2}$.

Clearly the Hamiltonian $H$ depends on the six real parame-

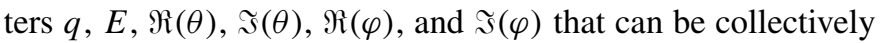
denoted by $R$. As $q$ and $E$ do not enter the expression for the biorthonormal system, the condition that $H$ be quasi-stationary only restricts $\theta$ and $\varphi$. Inserting (19) and (20) in (13) and simplifying the resulting equations, we can express this condition in the form of the following system of ordinary differential equations:

$\Im\left[\sin ^{2}\left(\frac{\theta^{*}}{2}\right) \dot{\varphi}\right]+\dot{v}_{1}=0$,

$\Im\left[\cos ^{2}\left(\frac{\theta^{*}}{2}\right) \dot{\varphi}\right]+\dot{v}_{2}=0$,

$\Im(\dot{\theta})-\mu \Re[\sin (\theta) \dot{\varphi}]=0$,

$\mu \Re(\dot{\theta})+\Im[\sin (\theta) \dot{\varphi}]=0$,

where $v_{a}:=\ln \left|\mathfrak{n}_{a}\right|$ for $a \in\{1,2\}$ and $\mu:=\frac{\left|\mathfrak{n}_{1}\right|^{2}-\left|\mathfrak{n}_{2}\right|^{2}}{\left|\mathfrak{n}_{1}\right|^{2}+\left|\mathfrak{n}_{2}\right|^{2}}$. Eqs. (22) and (23) may be solved to express $\mathfrak{n}_{1}$ and $\mathfrak{n}_{2}$ in terms of $\theta$ and $\varphi$. Substituting the result in Eqs. (24) and (25) yields two real equations for the four unknown functions $\Re(\theta)$, $\Im(\theta), \Re(\varphi)$ and $\Im(\varphi)$. Note that Eqs. (22)-(25) are timereparameterization-invariant; we can eliminate $t$ from these equations and express them in terms of any of the real parameters of the system, e.g., $\Re(\varphi)$.

By construction, solving (22)-(25) is equivalent to demanding that $\eta_{+}$as given by (21) is constant. This means that both $H(0)$ and $H(t)$ (for any $t \in[0, T]$ ) are $\eta_{+}$-pseudo-Hermitian. Therefore, we can obtain a characterization of quasi-stationary Hamiltonians $H(t)$ by setting $t=0$ in (21) and finding the form of $H(t)$ that is $\eta_{+}$-pseudo-Hermitian. This allows for an algebraic solution of the system of Eqs. (22)-(25).

The problem of finding the general form of an $\eta_{+}$-pseudoHermitian operator for metric operators of the form (21) has also been solved in [23]. Here we summarize the result. There are two possibilities:

(1) $\lambda(0)=0$ (i.e., $\eta_{+}$is diagonal), which corresponds to the cases: (1.a) $\theta(0)=0$; (1.b) $\theta(0) \in \mathbb{R}$ and $u=1$. For theses cases, we have

$\mathfrak{\Im}[\mathfrak{a}(t)]=0$,

$\mathfrak{c}(t)=\left[\frac{a(0) u+b(0)}{a(0)+b(0) u}\right] e^{-2 \Im[\varphi(0)]} \mathfrak{b}(t)^{*}$.

Here $q(t), \Re[\mathfrak{a}(t)], \mathfrak{R}[\mathfrak{b}(t)]$ and $\Im[\mathfrak{b}(t)]$ are arbitrary realvalued functions.
(2) $\lambda(0) \neq 0$ (i.e., $\eta_{+}$is not diagonal), which corresponds to the cases: (2.a) $u \neq 1$ and $\theta(0) \neq 0$; (2.b) $\theta(0) \notin \mathbb{R}$. For theses cases, we have

$$
\begin{aligned}
& \mathfrak{b}(t)=\lambda(0)^{-1}\{f(t)+i r \Im[\mathfrak{a}(t)]\}, \\
& \mathfrak{c}(t)=\left[r \lambda(0)^{*}\right]^{-1}\left\{s f(t)-2|\lambda(0)|^{2} \Re[\mathfrak{a}(t)]-i r s \Im[\mathfrak{a}(t)]\right\},
\end{aligned}
$$

where $r:=e^{2 \Im[\varphi(0)]}[a(0)+b(0) u], s:=a(0) u+b(0)$, and $f$ is an arbitrary real-valued function. Again, $H$ has four functional real degrees of freedom, namely $q(t), f(t), \Re[\mathfrak{a}(t)]$ and $\Im[\mathfrak{a}(t)]$.

Note that in both cases $u$ is a positive real constant that can sometimes be determined by setting $t=0$ in (26)-(28). If this fixes $u$, the metric operator $\eta_{+}$is uniquely determined up to the unimportant multiplicative constant $k$. Otherwise, similarly to the case of a time-independent Hamiltonian, the determination of $\eta_{+}$amounts to making a choice for $u$, [23]. We will return to this problem in Section 4.

In practice we can employ the above results as follows. Given a (non-diagonal) time-dependent $2 \times 2$ matrix Hamiltonian $H(t)$, we determine whether it is diagonalizable and has a real spectrum by examining its trace and the determinant of its traceless part [16]. If both of these quantities are real and the latter is negative, $H(t)$ is diagonalizable and has a real spectrum. ${ }^{10}$ But as we explained in the preceding section, this is not sufficient for formulating a consistent quantum theory using $H(t)$. In addition, the Hamiltonian must be quasi-stationary. To see if this is the case we examine its diagonal entries. If they are both real, then the Hamiltonian is quasi-stationary if (26) holds with $u=1$. If at least one of the diagonal entries is not real, then the Hamiltonian is quasi-stationary provided that it satisfies (27) and (28).

\section{Uniqueness of the metric operator}

For a time-independent diagonalizable Hamiltonian $H$ with a real spectrum, the metric operator $\eta_{+}$that makes $H, \eta_{+^{-}}$ pseudo-Hermitian is not unique $[16,19]$. In general one must fix a metric operator $\eta_{+}$directly [5] or alternatively select a set of so-called compatible irreducible operators and demand that all of these operators be $\eta_{+}$-pseudo-Hermitian [1,23]. The latter will fix $\eta_{+}$up to an unimportant multiplicative positive real constant.

The situation is different for a time-dependent Hamiltonian. The requirement that a generic time-dependent Hamiltonian $H(t)$ be quasi-stationary, i.e., $H(t)$ be $\eta_{+}$-pseudo-Hermitian for a constant $\eta_{+}$, will fix $\eta_{+}$(again up to an unimportant multiplicative positive constant). To see this, consider a general quasi-stationary Hamiltonian $H(t)$ and suppose that $\eta_{+}$ is a constant metric operator such that $H(t)$ is $\eta_{+}$-pseudoHermitian. Then, for all $t \in[0, T]$,

$H(t)^{\dagger}=\eta_{+} H(t) \eta_{+}^{-1}$.

$\overline{10}$ In this case $H(t)$ can be put in the form (17). 
Setting $t=0$ in this relation implies that $H(0)$ is $\eta_{+}$-pseudoHermitian. Differentiating both sides of (29) successively, setting $t=0$, and defining $O_{0}=H(0)$ and for all $n \in \mathbb{Z}^{+}$, $O_{n}:=\left.\frac{d^{n}}{d t^{n}} H(t)\right|_{t=0}$, we find that $\left(O_{0}, O_{1}, O_{2}, \ldots\right)$ is an infinite sequence of $\eta_{+}$-pseudo-Hermitian operators. Assuming that $O_{n}$ 's do not share a common eigenvector, which is true for a generic Hamiltonian $H(t)$, the sequence $\left(O_{0}, O_{1}, O_{2}, \ldots\right)$ includes among its terms an irreducible set of operators. Therefore, according to the uniqueness theorem proven in [1], $\eta_{+}$is unique up to a constant factor. For the two-dimensional systems considered in Section 3, this manifests itself through the fact that for a generic Hamiltonian the parameter $u$ that represents the arbitrariness in the choice of $\eta_{+}$is fixed by setting $t=0$ in Eqs. (26)-(28).

To see how this is done, consider the case that $q=0$ and $\theta=\frac{\pi}{2}$, i.e.,

$H(t)=\left(\begin{array}{cc}0 & \mathfrak{b}(t) \\ \mathfrak{c}(t) & 0\end{array}\right)$.

Then the condition that $H(t)$ is a (nonzero) diagonalizable operator with a real spectrum takes the form $\mathfrak{b} c \in \mathbb{R}^{+}$. To obtain the form of $\mathfrak{b}$ and $\mathfrak{c}$ for which $H(t)$ is quasi-stationary, we consider the following two possibilities:

(i) $u=1$ : In this case $\lambda(0)=0$ and we should enforce (26). But we can easily check that this does not put any restriction on $\mathfrak{b}$ and $\mathfrak{c}$.

(ii) $u \neq 1$ : In this case $\lambda(0) \neq 0$ and we should enforce (27) and (28). They give

$\mathfrak{b}=\frac{2 e^{-i \varphi(0)} f(t)}{u_{0}-1}, \quad \mathfrak{c}=\frac{2 e^{i \varphi(0)} f(t)}{u_{0}-1}$,

where $u$ is an arbitrary positive real number different from 1 , $\varphi(0)$ is an arbitrary complex number, and $f(t)$ is an arbitrary real-valued function. Note that according to (31),

$H(t)=f(0)^{-1} f(t) H(0)$.

This is the trivial case, where the eigenvectors of $H(t)$ happen to be time-independent.

The above analysis shows that if a Hamiltonian of the form (30) does not satisfy (32) for any real-valued function $f$, then we must choose $u=1$. This in turn means that the metric operator $\eta_{+}$is determined uniquely up to the constant factor $k$. But if we can satisfy (32) for some $f$, then $u$ may be chosen arbitrarily. In the latter case, similarly to the case of a timeindependent Hamiltonian in order to fix $\eta_{+}$, we must also make a choice for $u$.

\section{Concluding remarks}

In this Letter we have shown that unlike for a time-independent Hamiltonian operator, the conditions of diagonalizability and reality of the spectrum of a time-dependent Hamiltonian do not generally guarantee the unitarity of the corresponding timeevolution. The latter puts a further restriction on the choice of the Hamiltonian. We have examined the general form of this restriction, elucidated its geometric nature, and given a complete characterization of time-dependent $2 \times 2$ matrix Hamiltonians that define consistent quantum theories. We have also shown that, in contrast to the case of a time-independent Hamiltonian, a generic time-dependent Hamiltonian that is capable of defining a consistent quantum theory determines the metric operator and the inner product of the physical Hilbert space uniquely (up to a physically irrelevant multiplicative numerical factor).

\section{References}

[1] F.G. Scholtz, H.B. Geyer, F.J.W. Hahne, Ann. Phys. (N.Y.) 213 (1992) 74. [2] A. Mostafazadeh, J. Math. Phys. 43 (2002) 2814.

[3] C.M. Bender, D.C. Brody, H.F. Jones, Phys. Rev. Lett. 89 (2002) 270401.

[4] A. Mostafazadeh, J. Phys. A 36 (2003) 7081; A. Mostafazadeh, Czech. J. Phys. 54 (2004) 1125.

[5] A. Mostafazadeh, A. Batal, J. Phys. A 37 (2004) 11645; A. Mostafazadeh, J. Phys. A 38 (2005) 3213.

[6] C.M. Bender, I. Cavero-Pelaez, K.A. Milton, K.V. Shajesh, Phys. Lett. B 613 (2005) 97;

C.M. Bender, H.F. Jones, R.J. Rivers, Phys. Lett. B 625 (2005) 333, and references therein.

[7] A. Mostafazadeh, Class. Quantum Grav. 20 (2003) 155; A. Mostafazadeh, Ann. Phys. (N.Y.) 309 (2004) 1.

[8] C. Figueira de Morisson, A. Fring, J. Phys. A 39 (2006) 9269.

[9] A. Mostafazadeh, J. Math. Phys. 43 (2002) 205.

[10] A. Mostafazadeh, Czech. J. Phys. 56 (2006) 919.

[11] A. Mostafazadeh, J. Math. Phys. 46 (2005) 102108; A. Mostafazadeh, J. Phys. A 39 (2006) 13495.

[12] W. Pauli, Rev. Mod. Phys. 15 (1943) 175.

[13] P.A.M. Dirac, Proc. R. Soc. London A 180 (1942) 1.

[14] K.L. Nagy, State Vector Spaces with Indefinite Metric Quantum Field Theory, Noordhoff, Groningen, Netherlands, 1966; N. Nakanishi, Suppl. Prog. Theor. Phys. 51 (1972) 1.

[15] T.Ya. Azizov, I.S. Iokhvidov, Linear Operators in Spaces with Indefinite Metric, Wiley, Chichester, 1989.

[16] A. Mostafazadeh, Nucl. Phys. B 640 (2002) 419.

[17] C.M. Bender, D.C. Brody, H.F. Jones, Phys. Rev. D 70 (2004) 025001.

[18] M. Znojil, H.B. Geyer, Phys. Lett. B 640 (2006) 52.

[19] A. Mostafazadeh, J. Math. Phys. 44 (2003) 974; A. Mostafazadeh, J. Phys. A 39 (2006) 10171.

[20] H.B. Geyer, F.G. Scholtz, I. Snyman, Czech. J. Phys. 54 (2004) 1069; H.F. Jones, J. Mateo, Phys. Rev. D 73 (2006) 085002; D.P. Musumbu, H.B. Geyer, W.D. Heiss, J. Phys. A 40 (2007) F75.

[21] T. Kato, Perturbation Theory for Linear Operators, Springer, Berlin, 1995.

[22] J.C. Garrison, E.M. Wright, Phys. Lett. A 128 (1988) 177.

[23] A. Mostafazadeh, S. Özçelik, Turk. J. Phys. 30 (2006) 437.

[24] C.M. Bender, P.N. Meisinger, Q. Wang, J. Phys. A 36 (2003) 6791; A. Mostafazadeh, J. Math. Phys. 45 (2004) 932;

Y. Ben-Aryeh, A. Mann, I. Yaakov, J. Phys. A 37 (2004) 12059; A.D. Dutra, M.B. Hott, V.G.C.S. dos Santos, Europhys. Lett. 71 (2005) 166

P.K. Ghosh, J. Phys. A 38 (2005) 7313.

[25] A. Kvitsimsky, S. Putterman, J. Math. Phys. 32 (1991) 1403. 